\title{
Long non-coding RNA MALAT1 serves as an independent predictive biomarker for the diagnosis, severity and prognosis of patients with sepsis
}

\author{
JIANJUN CHEN $^{1 *}$, YINGFENG HE $^{1 *}$, LIANGLIANG ZHOU ${ }^{1 *}$, YIJUN DENG $^{1}$ and LINJIE SI ${ }^{2}$ \\ ${ }^{1}$ Department of Intensive Care Medicine, The First People's Hospital of Yancheng, Yancheng, Jiangsu 224000; \\ ${ }^{2}$ Department of Cardiovascular Surgery, The First Affiliated Hospital of Nanjing Medical University, \\ Nanjing, Jiangsu 210029, P.R. China
}

Received March 17, 2019; Accepted October 3, 2019

DOI: $10.3892 / \mathrm{mmr} .2020 .10923$

\begin{abstract}
The present prospective study was conducted to investigate the independent risk and predictive value of plasma long non-coding RNA metastasis-associated lung adenocarcinoma transcript 1 (MALAT1) as a biomarker for the diagnosis, severity and prognosis of sepsis. A total of 120 patients with sepsis and 60 healthy controls (HCs) were recruited. The expression levels of plasma MALAT1 were detected by quantitative PCR. The results demonstrated that the plasma levels of MALAT1 were significantly increased in patients with sepsis compared with $\mathrm{HCs}(\mathrm{P}<0.001)$, in patients with septic shock compared with in patients without septic shock $(\mathrm{P}<0.001)$, and in non-survivors compared with in survivors $(\mathrm{P}<0.001)$. MALAT1 plasma levels exhibited weak positive correlation with serum procalcitonin levels $(\mathrm{r}=0.253 ; \mathrm{P}=0.005)$, arterial lactate levels $(\mathrm{r}=0.488 ; \mathrm{P}<0.001)$, sepsis-related organ failure assessment scores $(\mathrm{r}=0.566 ; \mathrm{P}<0.001)$, and acute physiology and chronic health evaluation II scores $(r=0.517 ; \mathrm{P}<0.001)$ in patients with sepsis. Multivariate logistic regression analysis revealed that high MALAT1 expression was an independent risk factor for sepsis $(\mathrm{P}<0.001)$, septic shock $(\mathrm{P}=0.030)$ and poor prognosis $(\mathrm{P}=0.015)$. In addition, the receiver operating characteristic curve exhibited a significant predictive value for MALAT1 in distinguishing patients with sepsis from HCs with an area under the curve (AUC) of 0.910, patients with septic shock from patients without shock with an AUC of 0.836, and non-survivors from survivors with an AUC of 0.886. In
\end{abstract}

Correspondence to: Dr Linjie Si, Department of Cardiovascular Surgery, The First Affiliated Hospital of Nanjing Medical University, 300 Guangzhou Road, Nanjing, Jiangsu 210029, P.R. China E-mail: silinjie001@163.com

*Contributed equally

Key words: long non-coding RNA, metastasis-associated lung adenocarcinoma transcript 1 , sepsis, diagnosis, prognosis conclusion, plasma MALAT1 may serve as a biomarker for the diagnosis, severity and prognosis of sepsis.

\section{Introduction}

Sepsis is defined as life-threatening organ dysfunction caused by an abnormal host response to infection, and septic shock is a subset of sepsis (1). Clinically, organ dysfunction can be represented by an increase in the sepsis-related organ failure assessment (SOFA) score of 2 points or more; septic shock can be identified by a vasopressor requirement to maintain mean arterial pressure $\geq 65 \mathrm{mmHg}$ and a serum lactate level $>2 \mathrm{mmol} / \mathrm{l}$ in the absence of hypovolemia (2). Sepsis has a high mortality rate and is one of the main causes of death in intensive care units (ICUs) worldwide (3). Despite recent progress in the development of anti-infective drugs and life support treatments, the mortality associated with sepsis, particularly septic shock, is still high (4). The pathogenesis of sepsis is complicated and has not yet been fully elucidated (5). The major pathophysiological mechanisms underlying sepsis include systemic inflammation, immune imbalance, multiple organ dysfunction and gene polymorphism (6). Early diagnosis and intervention are the most effective methods to reduce the mortality rates of patients with sepsis (7). The severity and prognosis of sepsis are determined by procalcitonin (PCT) and lactate (Lac) levels, SOFA (2), and acute physiology and chronic health evaluation II (APACHE II) (8). However, there is still a lack of precise biomarkers and intervention targets for sepsis.

Advances in human genome-wide analysis have revealed that $2 \%$ of the genome is composed of protein-coding genes, whereas the remaining $98 \%$ of genome transcripts serve no protein-coding functions (9). Long non-coding RNAs (lncRNAs) are a class of non-protein-coding RNAs with a length of $>200$ nucleotides that are widely involved in cell proliferation, differentiation and apoptosis through regulating target gene expression (10). The IncRNA metastasis-associated lung adenocarcinoma transcript 1 (MALAT1) was first identified in non-small cell lung cancer (11) and is among the most studied lncRNAs (12). MALAT1 is involved in a variety of physiological and pathological processes, 
including neurodevelopment (13), skeletal myogenesis (14), angiogenesis (15), cancer (16), and cardiovascular (17) and neurological (18) diseases. Recent studies have reported that MALAT1 is also associated with infection and immune-mediated inflammatory diseases, such as sepsis (19), systemic lupus erythematosus (20), multiple sclerosis (21) and rheumatoid arthritis (22), by regulating the inflammatory response through multiple target genes and signaling pathways. However, the clinical value of MALAT1 in the diagnosis, severity and prognosis of sepsis has not been reported. Therefore, the aim of the present study was to determine the expression levels of plasma MALAT1 in patients with sepsis and to evaluate whether MALAT1 may serve as an independent predictive biomarker for patients with sepsis.

\section{Materials and methods}

Participants. A total of 120 patients with sepsis were admitted to the ICU of The First People's Hospital of Yancheng between June 2016 and June 2018, and were consecutively recruited for this prospective cohort study. The management of sepsis and septic shock was performed in accordance with the Surviving Sepsis Campaign: International Guidelines for Management of Sepsis and Septic Shock (1). The inclusion criteria for the present study were as follows: i) Patients who met the aforementioned diagnostic criteria for sepsis and/or septic shock; ii) patients aged between 18 and 80 years; and iii) the primary causes of sepsis and septic shock were pulmonary infection, abdominal infection, urinary tract infection and/or bacteremia. Patients $<18$ or $>80$ years, pregnant and/or lactating patients, those with tumors, blood disease, organ dysfunction and immune-mediated inflammatory disease were excluded. During the same period, 60 healthy volunteers with matching age and sex were recruited as healthy controls (HCs). The inclusion criteria were as follows: i) Subjects aged between 18 and 80 years; and ii) subjects with good physical and mental health. HCs with a history of immune-mediated inflammatory disease, severe infection, organ dysfunction, cancer, or immunosuppressive therapy were excluded. HCs who were pregnant and/or lactating at the time of recruitment were also excluded.

This study was approved by the Ethics Committee of The First People's Hospital of Yancheng and complied with the ethics standards of the Declaration of Helsinki. All participants or their statutory guardians provided written informed consent.

Study design. Firstly, the expression levels of MALAT1 in plasma samples from $\mathrm{HCs}$ and patients with sepsis were analyzed and compared between patients with sepsis and $\mathrm{HCs}$, patients with and without septic shock, as well as survivors and non-survivors. Subsequently, the correlation between MALAT1 expression levels and conventional evaluation indicators of sepsis, including PCT and Lac levels, as well as SOFA and APACHE II scores, was evaluated. Multivariate logistic regression was used to analyze whether MALAT1 may serve as an independent risk factor for the diagnosis, severity and prognosis of sepsis. Finally, the predictive values of MALAT1 in distinguishing patients with sepsis from HCs, patients with septic shock from those without septic shock, and non-survivors from survivors were evaluated.
Blood sample preparation. The blood samples of all subjects were collected within $24 \mathrm{~h}$ of admission. Elbow venous blood $(10 \mathrm{ml} / \mathrm{subject})$ was divided into two parts: One $(5 \mathrm{ml})$ was collected into a vacutainer tube containing EDTA (Becton, Dickinson and Company) for plasma separation; the other part $(5 \mathrm{ml})$ was collected into a vacutainer tube containing clot activator and polymer gel (Becton, Dickinson and Company) for serum separation. To obtain plasma/serum, the whole blood was centrifuged at $2,500 \mathrm{xg}$ for $15 \mathrm{~min}$ at $4^{\circ} \mathrm{C}$. All samples were stored at $-80^{\circ} \mathrm{C}$ until further processing.

Data collection. The clinical data of patients with sepsis were collected within $24 \mathrm{~h}$ following admission, and included age, sex, serum PCT levels, arterial Lac levels, SOFA and APACHE II scores. Patients were assessed by a senior physician for sepsis severity (septic shock or no shock). The prognosis of patients with sepsis for 28 days following admission (survival or non-survival) was also recorded. Age, sex and PCT levels were recorded in HCs. The serum PCT levels were detected by chemiluminescent immunoassay (Wuhan EasyDiagnosis Biomedicine Co., Ltd.; cat. no. ED75440-Hu) according to the manufacturer's protocol. Lac levels were detected by arterial blood gas analysis.

Reverse transcription-quantitative PCR (RT-qPCR). Total RNA was extracted from isolated plasma using TRIzol ${ }^{\circledR}$ reagent (Invitrogen; Thermo Fisher Scientific, Inc.) and was reverse transcribed into cDNA using the PrimeScript ${ }^{\mathrm{TM}} \mathrm{RT}$ reagent kit (Takara Bio, Inc.), according to the manufacturer's protocol. Subsequently, the expression levels of MALAT1 were determined using SYBR Premix Ex Taq ${ }^{\mathrm{TM}}$ II (Takara Bio, Inc.) on the ABI Prism 7300 RT-qPCR system (Applied Biosystems; Thermo Fisher Scientific, Inc.). PCR was performed under the following conditions: Denaturation at $50^{\circ} \mathrm{C}$ for $2 \mathrm{~min}$, followed by 40 cycles of $95^{\circ} \mathrm{C}$ for $15 \mathrm{sec}$ and $60^{\circ} \mathrm{C}$ for $1 \mathrm{~min}$. The relative expression levels of MALAT1 were calculated using the $2^{-\Delta \Delta \mathrm{Cq}}$ method (23) and GAPDH was used as internal reference. The primer sequences used were as follows: MALAT1, forward 5'-GTGATGCGAGTTGTTCTCCG-3' reverse 5'-CTGGCT GCCTCAATGCCTAC-3'; and GAPDH, forward 5'-GAGTCA ACGGATTTGGTCGT-3' and reverse 5'-TTGATTTTGGAG GGATCTCG-3'.

Statistical analysis. Data are expressed as the mean \pm standard deviation, median and interquartile range or count. PASW Statistics 18.0 (SPSS Inc.) and GraphPad Prism 8.0 (GraphPad Software, Inc.) were used for statistical analysis. The comparisons between two groups were performed using unpaired Student's t-test, Mann-Whitney test or $\chi^{2}$ test as appropriate. Spearman's correlation was used to analyze the correlation between plasma MALAT1 and PCT, Lac, SOFA and APACHE II scores. Multivariate logistic regression and receiver operating characteristic (ROC) curve analyses were performed to determine the predictive value of independent risk factors for the diagnosis, severity and prognosis of sepsis. Risk factors with $\mathrm{P}<0.15$ from the univariate analyses (Student's t-test, Mann-Whitney test and $\chi^{2}$ test) were included in the multivariate logistic regression analysis. $\mathrm{P}<0.05$ was considered to indicate a statistically significant difference. 
Table I. Univariate analysis of clinicopathological characteristics between HCs and patients with sepsis.

\begin{tabular}{lccc}
\hline Factor & HCs $(\mathrm{n}=60)$ & Sepsis $(\mathrm{n}=120)$ & P-value \\
\hline Age, years & $47.68 \pm 10.25$ & $50.35 \pm 8.47$ & $0.085^{\mathrm{a}}$ \\
Sex, $\mathrm{n}$ & & & $0.082^{\mathrm{b}}$ \\
Male & 36 & 64 & \\
Female & 24 & $5.65(0.68-12.28)$ & $<0.001^{\mathrm{c}}$ \\
PCT, ng/ml & $0.30(0.14-0.40)$ & $3.39(2.22-5.83)$ & - \\
Lac, mmol/l & - & $7.38 \pm 3.89$ & - \\
SOFA score & - & $15.21 \pm 4.69$ & - \\
APACHE II score & - & & \\
\hline
\end{tabular}

${ }^{a}$ Student's t-test; ${ }^{\mathrm{b}} \chi^{2}$ test; ${ }^{\mathrm{c}}$ Mann-Whitney test. Data are presented as the mean \pm standard deviation, median and interquartile range or count. APACHE II, acute physiology and chronic health evaluation II; HCs, healthy controls; Lac, lactate; PCT, procalcitonin; SOFA, sepsis-related organ failure assessment.

\section{Results}

MALAT1 as an independent risk factor for sepsis. Univariate analysis revealed that age $(\mathrm{P}=0.085)$ and sex $(\mathrm{P}=0.082)$ were not significantly different between patients with sepsis and $\mathrm{HCs}$ (Table I). However, PCT $(\mathrm{P}<0.001)$ and MALAT1 $(\mathrm{P}<0.001)$ levels were significantly higher in patients with sepsis compared with those in HCs (Fig. 1A). In addition, multivariate logistic regression analysis revealed that high MALAT1 expression $(\mathrm{P}<0.001)$ and PCT $(\mathrm{P}=0.007)$ levels were independent predictive factors for sepsis risk, whereas, age $(\mathrm{P}=0.161)$ and sex $(\mathrm{P}=0.084)$ exhibited no association with sepsis risk (Table II).

Diagnostic value of MALAT1 for sepsis. ROC curve analysis revealed that MALAT1 had significant diagnostic value for sepsis with an area under curve (AUC) of 0.910 (Fig. 1B); however, the diagnostic value of MALAT1 was lower compared with that of PCT (AUC=0.928; Fig. 1C). The optimal cut-off point for the level of MALAT1 that distinguished patients with sepsis from HCs was $>1.895$, and the specificity and sensitivity were 85 and $83.33 \%$, respectively. In addition, the optimal cut-off point for PCT level that distinguished patients with sepsis from $\mathrm{HCs}$ was $<0.5$, and the specificity and sensitivity were 93.33 and $81.67 \%$, respectively.

Correlation between plasma MALAT1 levels and conventional evaluation indicators of sepsis. Spearman's correlation analysis revealed that MALAT1 plasma levels exhibited weak positive correlations with PCT levels ( $\mathrm{r}=0.253$; $\mathrm{P}=0.005)$, Lac levels $(\mathrm{r}=0.488 ; \mathrm{P}<0.001)$, SOFA scores $(\mathrm{r}=0.566 ; \mathrm{P}<0.001)$ and APACHE II scores $(\mathrm{r}=0.517 ; \mathrm{P}<0.001)$ in patients with sepsis (Fig. 2).

MALAT1 is an independent risk factor for septic shock. As presented in Table III, univariate analysis revealed that age $(\mathrm{P}=0.124)$, sex $(\mathrm{P}=0.912)$ and PCT level $(\mathrm{P}=0.257)$ were not significantly associated with the occurrence of septic shock. However, Lac levels $(\mathrm{P}<0.001)$, SOFA scores $(\mathrm{P}<0.001)$, APACHE II scores $(\mathrm{P}<0.001)$ and MALAT1 levels $(\mathrm{P}<0.001$; Fig. 3A) were significantly higher in patients with septic shock compared with in those without septic shock. In addition,
Table II. Multivariate logistic regression of risk factors for sepsis.

\begin{tabular}{lrrl}
\hline Factor & P-value & OR & \multicolumn{1}{c}{$95 \%$ CI } \\
\hline Age & 0.161 & 1.050 & $0.981-1.123$ \\
Sex (male vs. female) & 0.084 & 3.009 & $0.861-10.514$ \\
MALAT1 & $<0.001$ & 7.446 & $3.259-17.012$ \\
PCT & 0.007 & 12.233 & $1.960-76.353$ \\
\hline
\end{tabular}

CI, confidence interval; MALAT1, metastasis-associated lung adenocarcinoma transcript 1 ; OR, odds ratio; PCT, procalcitonin.

multivariate logistic regression analysis demonstrated that high MALAT1 $(\mathrm{P}=0.030)$ or Lac $(\mathrm{P}=0.027)$ levels, and SOFA $(\mathrm{P}=0.001)$ and APACHE II $(\mathrm{P}=0.011)$ scores were independent risk factors for septic shock, whereas age $(\mathrm{P}=0.368)$ exhibited no association with the severity of sepsis (Table IV).

Diagnostic value of MALAT1 level for septic shock. ROC curve analysis exhibited significant predictive value for MALAT1 (AUC=0.836) in distinguishing patients with septic shock from those without septic shock (Fig. 3B). This value was higher compared with that for Lac (AUC=0.830; Fig. 3C), but lower compared with those for SOFA (AUC=0.908; Fig. 3D) and APACHE II (AUC=0.856; Fig. 3E) scores. At the optimal cut-off point of $>3.665$ for MALAT1 levels, the specificity and sensitivity were 92.42 and $70.37 \%$, respectively. At the optimal cut-off point of $>3.215$ for Lac levels, the specificity and sensitivity were 74.24 and $81.48 \%$, respectively. At the optimal cut-off point of $>6$ for SOFA scores, the specificity and sensitivity were 83.33 and $87.04 \%$, respectively. At the optimal cut-off point of $>13$ for APACHE II scores, the specificity and sensitivity were 71.21 and $87.04 \%$, respectively.

MALAT1 is an independent risk factor for poor prognosis of sepsis. As presented in Table $\mathrm{V}$, univariate analysis revealed that age $(\mathrm{P}=0.496)$ and sex $(\mathrm{P}=0.939)$ were not significantly different between survivors and non-survivors. However, septic 

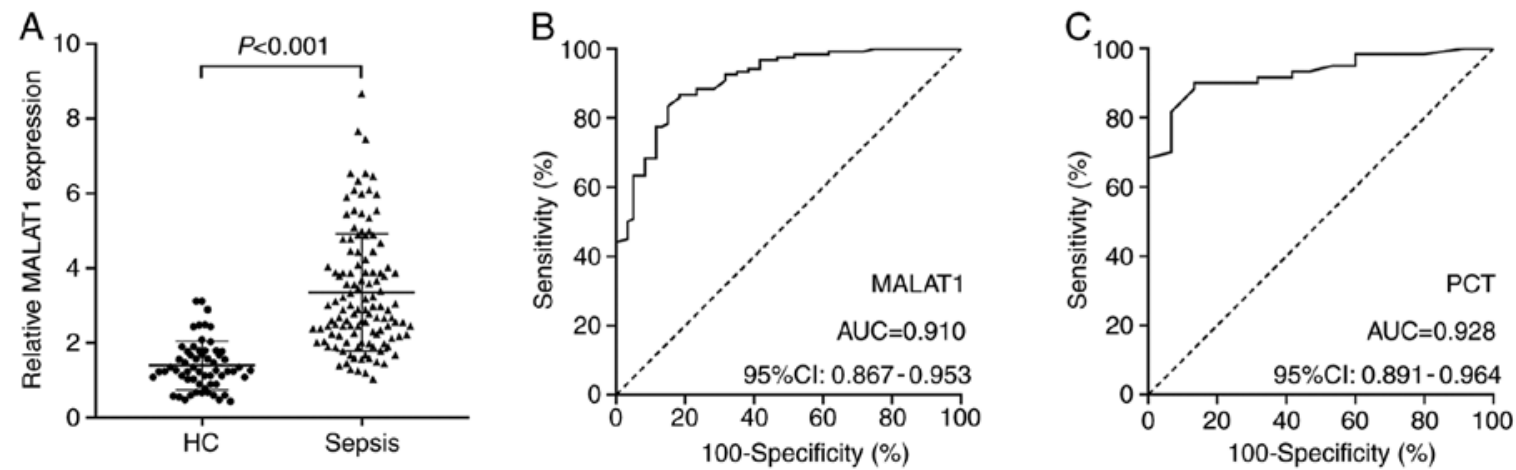

Figure 1. Relative expression and predictive value of MALAT1 plasma levels in distinguishing patients with sepsis from HCs. (A) Plasma MALAT1 expression was significantly increased in patients with sepsis compared with HCs. (B and C) Receiver operating characteristic curves for (B) MALAT1 and (C) PCT levels. CI, confidence interval; HC, healthy control; MALAT1, metastasis-associated lung adenocarcinoma transcript 1; PCT, procalcitonin.
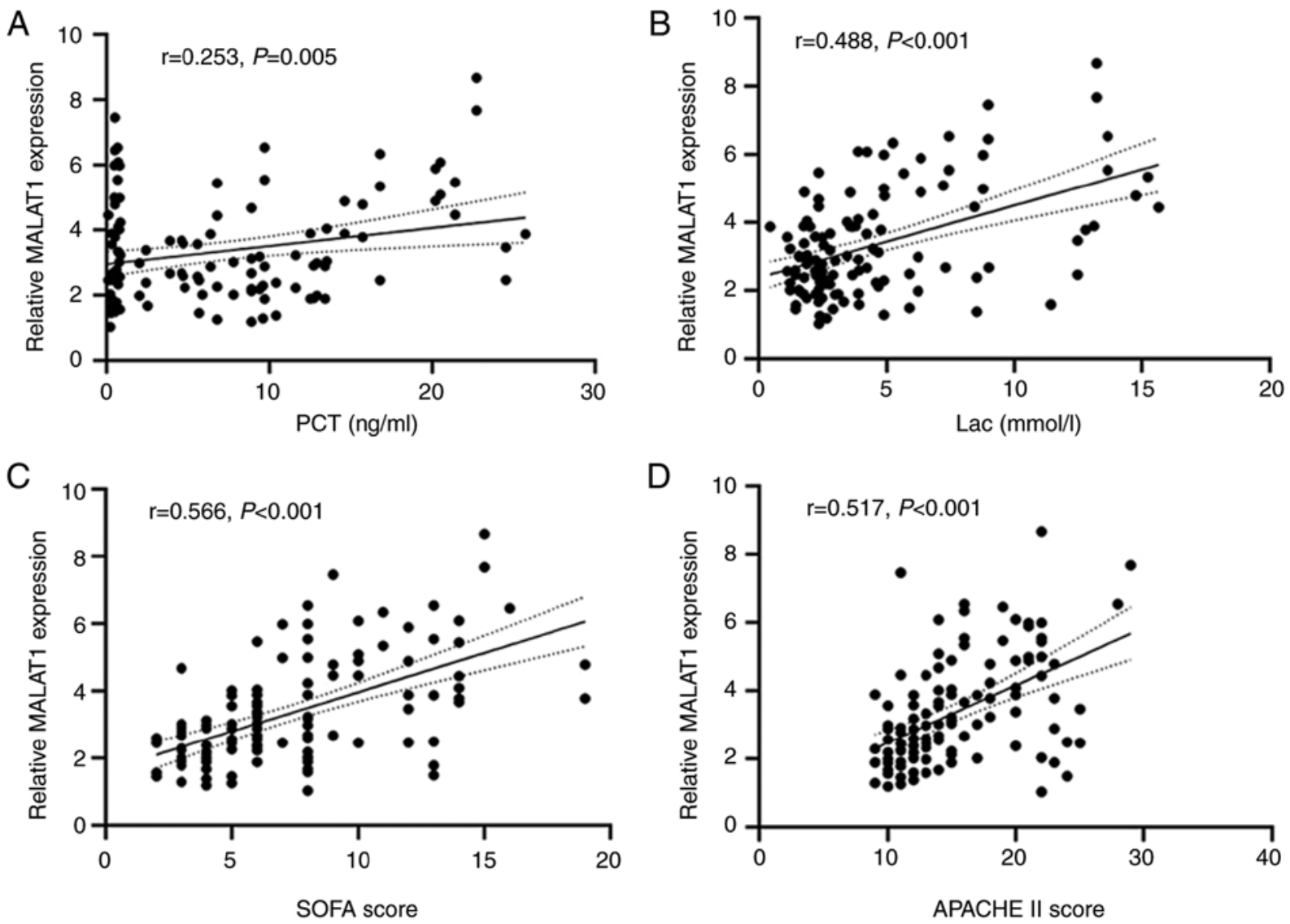

Figure 2. Correlation between MALAT1 plasma levels and conventional evaluation indicators of sepsis. (A-D) MALAT1 plasma levels exhibited weak positive correlations with (A) PCT and (B) Lac levels, as well as (C) SOFA and (D) APACHE II scores in patients with sepsis. APACHE II, acute physiology and chronic health evaluation II; Lac, lactate; MALAT1, metastasis-associated lung adenocarcinoma transcript 1; PCT, procalcitonin; SOFA, sepsis-related organ failure assessment.

shock $(\mathrm{P}<0.001)$, $\mathrm{PCT}$ levels $(\mathrm{P}=0.038)$, Lac levels $(\mathrm{P}<0.001)$, and SOFA $(\mathrm{P}<0.001)$ and APACHE II $(\mathrm{P}<0.001)$ scores, as well as MALAT1 plasma levels (Fig. 4A; $\mathrm{P}<0.001$ ) were significantly increased in non-survivors compared with survivors. Further multivariate logistic regression analysis demonstrated that high MALAT1 $(\mathrm{P}=0.015)$ and Lac $(\mathrm{P}=0.023)$ levels, SOFA $(\mathrm{P}=0.011)$ and APACHE II $(\mathrm{P}=0.028)$ scores, and septic shock $(\mathrm{P}=0.043)$ were all independent predictive factors for poor prognosis of sepsis, whereas PCT levels $(\mathrm{P}=0.156)$ were not associated with prognosis in patients with sepsis (Table VI).
Prognostic value of MALAT1 level for sepsis. ROC curve analysis exhibited significant predictive value for MALAT1 levels (AUC $=0.886)$ at distinguishing non-survivors from survivors (Fig. 4B), which was higher compared with Lac levels (AUC $=0.868$; Fig. 4C) and APACHE II scores (AUC=0.868; Fig. 4E), but lower compared with SOFA scores (AUC=0.943; Fig. 4D). At the optimal cut-off point of $>3.62$ for MALAT1 levels, the specificity and sensitivity were 89.47 and $81.82 \%$, respectively. At the optimal cut-off point of $>3.51$ for Lac levels, the specificity and sensitivity were 75 and 
Table III. Univariate analysis of clinicopathological characteristics between patients with and without septic shock.

\begin{tabular}{lccc}
\hline Factor & No septic shock $(\mathrm{n}=66)$ & Septic shock $(\mathrm{n}=54)$ & P-value \\
\hline Age, years & $49.27 \pm 8.90$ & $51.67 \pm 7.80$ & $0.124^{\mathrm{a}}$ \\
Sex, $\mathrm{n}$ & & & \\
Male & 30 & 24 & $0.912^{\mathrm{b}}$ \\
Female & 36 & 30 & $0.257^{\mathrm{c}}$ \\
PCT, ng/ml & $5.90(2.00-10.40)$ & $5.07(3.54-8.98)$ & $<0.001^{\mathrm{c}}$ \\
Lac, mmol/l & $2.34(1.72-3.72)$ & $10.31 \pm 3.51$ & $<0.001^{\mathrm{c}}$ \\
SOFA score & $4.97 \pm 2.13$ & $18.28 \pm 4.57$ & $<0.001^{\mathrm{c}}$ \\
APACHE II score & $12.70 \pm 3.13$ & & \\
\hline
\end{tabular}

${ }^{a}$ Student's t-test; ${ }^{b} \chi^{2}$ test; ${ }^{c}$ Mann-Whitney test. Data are presented as the mean \pm standard deviation, median and interquartile range or count. APACHE II, acute physiology and chronic health evaluation II; HCs, healthy controls; Lac, lactate; PCT, procalcitonin; SOFA, sepsis-related organ failure assessment.

Table IV. Multivariate logistic regression of risk factors for septic shock.

\begin{tabular}{lccc}
\hline Factor & P-value & OR & $95 \%$ CI \\
\hline Age, years & 0.368 & 1.036 & $0.959-1.119$ \\
MALAT1 & 0.030 & 2.030 & $1.070-3.851$ \\
Lac & 0.027 & 1.405 & $1.039-1.901$ \\
SOFA & 0.001 & 1.580 & $1.196-2.086$ \\
APACHE II & 0.011 & 1.268 & $1.057-1.522$ \\
\hline
\end{tabular}

APACHE II, acute physiology and chronic health evaluation II; CI, confidence interval; Lac, lactate; MALAT1, metastasis-associated lung adenocarcinoma transcript 1; OR, odds ratio; SOFA, sepsis-related organ failure assessment.

$88.64 \%$, respectively. At the optimal cut-off point of $>6$ for SOFA scores, the specificity and sensitivity were 77.63 and $93.18 \%$, respectively. At the optimal cut-off point of $>15$ for APACHE II scores, the specificity and sensitivity were 86.84 and $77.27 \%$, respectively.

\section{Discussion}

MALAT1 is ubiquitously expressed in the majority of human tissues and body fluids, which suggests that MALAT1 may be a potential biomarker for disease diagnosis, prognosis and treatment (24). A large proportion of current studies focus on the underlying molecular mechanisms of MALAT1 function in the regulation of various pathophysiological processes $(12,25,26)$; however, few studies have been conducted to investigate the clinical application of MALAT1 as a biomarker $(21,27)$. To the best of our knowledge, the present study is the first to evaluate the independent risk and predictive value of MALAT1 as a biomarker for the diagnosis, severity and prognosis of sepsis.

MALAT1 is located on human chromosome 11q13 (28), and serves pivotal roles in the inflammatory regulation of sepsis and septic organ dysfunction; for instance, the downregulation of MALAT1 attenuated cardiac inflammation and microvascular endothelial cell injury in rats with sepsis by inhibiting the inflammatory response and apoptosis $(29,30)$. The knockdown of MALAT1 suppressed lipopolysaccharide (LPS)-induced acute kidney and lung injury by alleviating the inflammatory response through the microRNA-146a/NF- $\mathrm{B}$ pathway $(31,32)$. In addition, MALAT1 has been demonstrated to be upregulated in LPS-induced macrophages and chondrocytes, whereas the knockdown of MALAT1 enhanced LPS-induced inflammatory injury by increasing the expression levels of the tumor necrosis factor (TNF)- $\alpha$ and interleukin (IL)- 6 through negative regulation of the $\mathrm{NF}-\kappa \mathrm{B}$ pathway (33-35). These results indicated that MALAT1 may be a pro- or anti-inflammatory regulator in different tissues and cells induced by LPS, which may be associated with the imbalance in the immune system during septic injury. Similarly, the results of the present study demonstrated that MALAT1 plasma levels were significantly increased in patients with sepsis. Higher expression levels of MALAT1 were also observed in patients with septic shock and those who succumbed to sepsis compared with the respective control groups.

To determine whether MALAT1 was associated with the diagnosis, severity and prognosis of sepsis, the correlation between MALAT1 levels and conventional indicators of sepsis, including PCT and Lac levels, as well as SOFA and APACHE II scores, was further evaluated. PCT is ubiquitously expressed in parathyroid glands during bacterial infection and is likely to be induced by TNF- $\alpha$ and IL-6 (36). PCT has been used in the diagnosis of sepsis for decades; in the present study, PCT levels were identified as an independent risk and diagnostic factor for sepsis, and MALAT1 levels exhibited a weak positive correlation with PCT levels. Dahaba and Metzler (37) reported that PCT levels were closely correlated with the severity of sepsis and exhibited a significant predictive value for the prognosis of sepsis on day 6 following admission. However, PCT levels had no independent predictive value for the severity and prognosis of sepsis in the present study, which may be due to the smaller sample size and earlier time of PCT level analysis (within $24 \mathrm{~h}$ of admission). Arterial Lac levels, SOFA and APACHE II scores are commonly used to assess the severity and prognosis of sepsis $(2,8)$. In accordance with previous studies, the results of the present study demonstrated that high Lac levels, SOFA 
Table V. Univariate analysis of clinicopathological characteristics between survivors and non-survivors.

\begin{tabular}{lccr}
\hline Factor & Survivors $(\mathrm{n}=76)$ & Non-survivors (n=44) & P-value \\
\hline Age, years & $49.95 \pm 7.97$ & $51.05 \pm 9.33$ & $0.496^{\mathrm{a}}$ \\
Sex, $\mathrm{n}$ & & & $0.939^{\mathrm{b}}$ \\
Male & 34 & 20 & \\
Female & 42 & & \\
Septic shock, $\mathrm{n}$ & & & \\
No & 59 & 37 & $<0.001^{\mathrm{b}}$ \\
Yes & 17 & $8.25(0.67-20.20)$ & $<.038^{\mathrm{c}}$ \\
PCT, ng/ml & $5.20(0.70-9.40)$ & $6.11(3.97-11.60)$ & $<0.001^{\mathrm{c}}$ \\
Lac, mmol/1 & $2.37(1.77-3.70)$ & $11.16 \pm 3.25$ & $<0.001^{\mathrm{c}}$ \\
SOFA score & $5.18 \pm 2.15$ & $18.98 \pm 4.33$ & $<0.001^{\mathrm{c}}$ \\
APACHE II score & $13.03 \pm 3.31$ & &
\end{tabular}

${ }^{a}$ Student's t-test; ${ }^{b} \chi^{2}$ test; ${ }^{c}$ Mann-Whitney test. Data are presented as the mean \pm standard deviation, median and interquartile range or count. APACHE II, acute physiology and chronic health evaluation II; HCs, healthy controls; Lac, lactate; PCT, procalcitonin; SOFA, sepsis-related organ failure assessment.
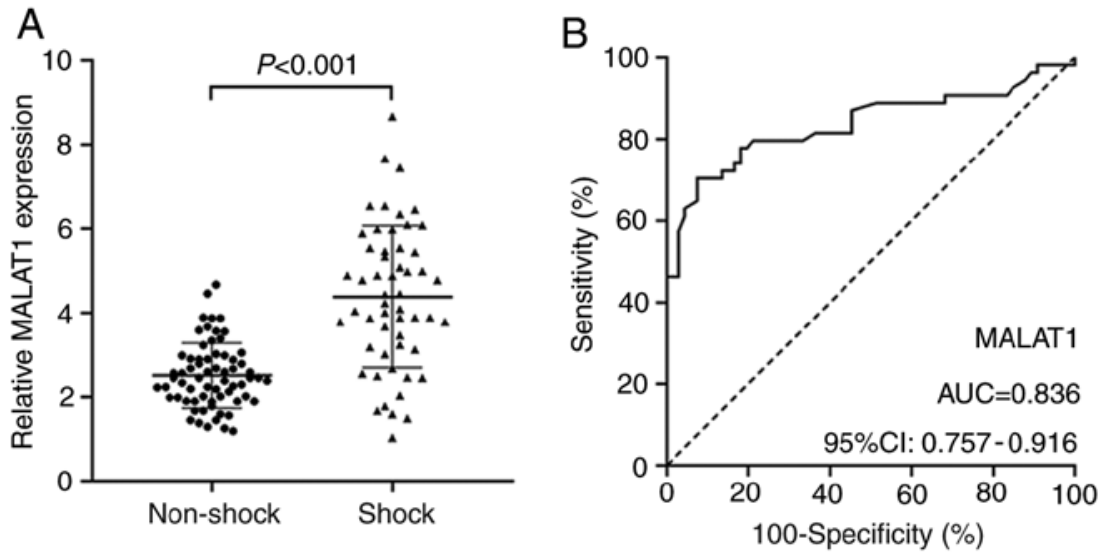

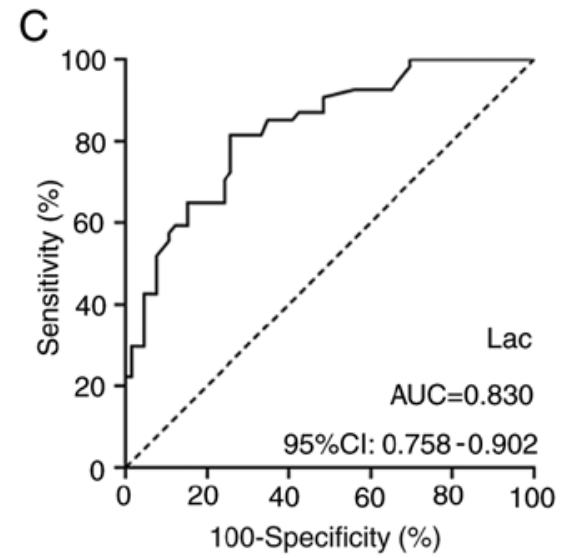

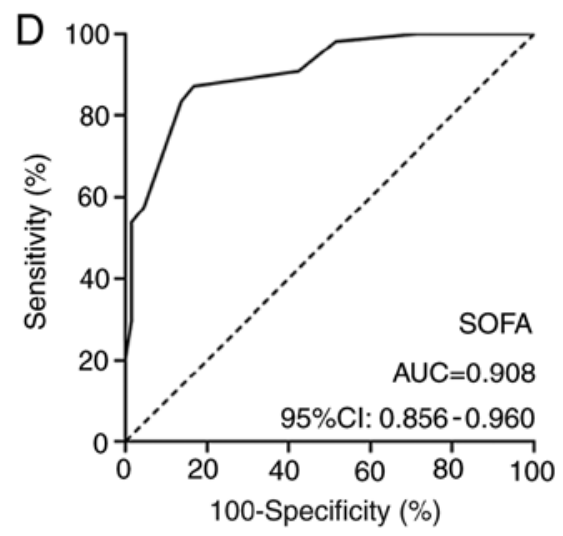

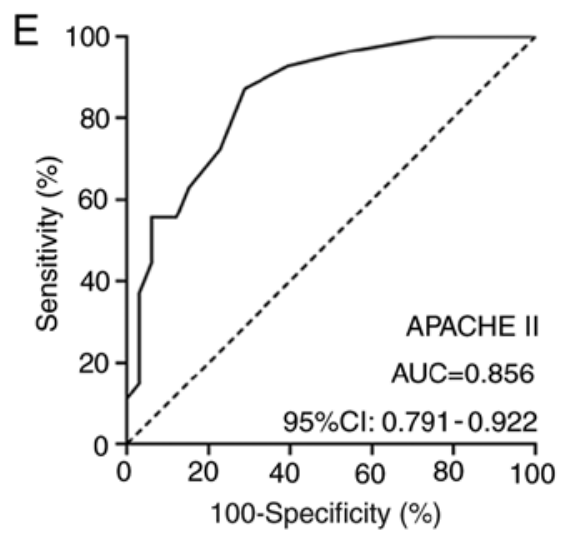

Figure 3. Relative expression and predictive value of MALAT1 plasma levels in distinguishing patients with septic shock from those without septic shock. (A) MALAT1 expression was significantly increased in patients with septic shock compared with those without septic shock. (B-E) Receiver operating characteristic curves for (B) MALAT1 and (C) Lac levels, (D) SOFA and (E) APACHE II scores. APACHE II, acute physiology and chronic health evaluation II; CI, confidence interval; Lac, lactate; MALAT1, metastasis-associated lung adenocarcinoma transcript 1; Non-shock, patients with sepsis without septic shock; PCT, procalcitonin; SOFA, sepsis-related organ failure assessment.

and APACHE II scores were independent predictive factors for the severity and mortality of sepsis. In addition, MALAT1 levels exhibited a weak positive correlation with Lac levels,
SOFA and APACHE II scores. These results suggested that plasma MALAT1 expression may be associated with the diagnosis, severity and prognosis of sepsis. 
Table VI. Multivariate logistic regression of mortality risk factors for sepsis.

\begin{tabular}{lccc}
\hline Factor & P-value & OR & $95 \%$ CI \\
\hline Septic shock (yes vs. no) & 0.043 & 50.144 & $1.133-2,219.541$ \\
MALAT1 & 0.015 & 3.819 & $1.303-11.189$ \\
PCT & 0.156 & 1.133 & $0.954-1.345$ \\
Lac & 0.023 & 1.933 & $1.096-3.410$ \\
SOFA & 0.011 & 4.054 & $1.379-11.917$ \\
APACHE II & 0.028 & 1.719 & $1.062-2.782$
\end{tabular}

APACHE II, acute physiology and chronic health evaluation II; CI, confidence interval; Lac, lactate; MALAT1, metastasis-associated lung adenocarcinoma transcript 1; OR, odds ratio; PCT, procalcitonin; SOFA, sepsis-related organ failure assessment.

A

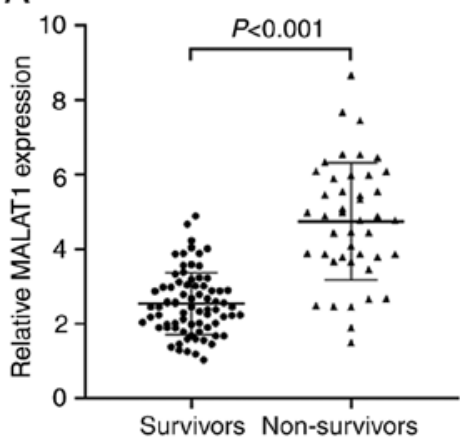

B

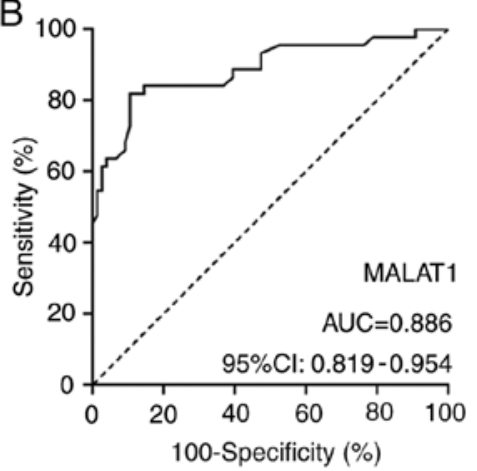

$\mathrm{C}$

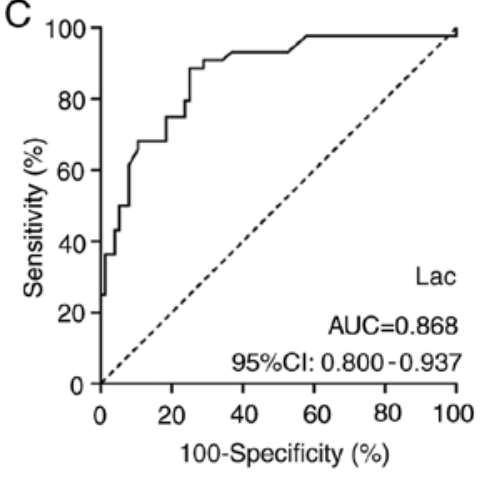

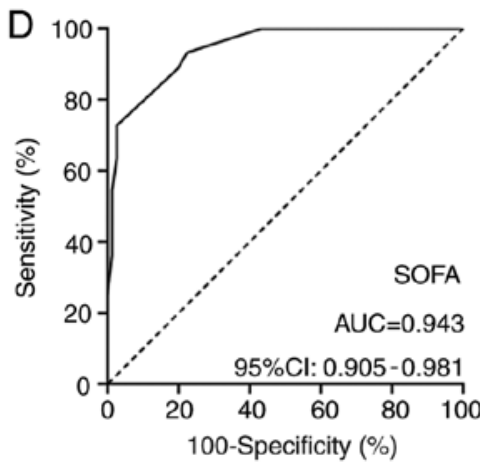

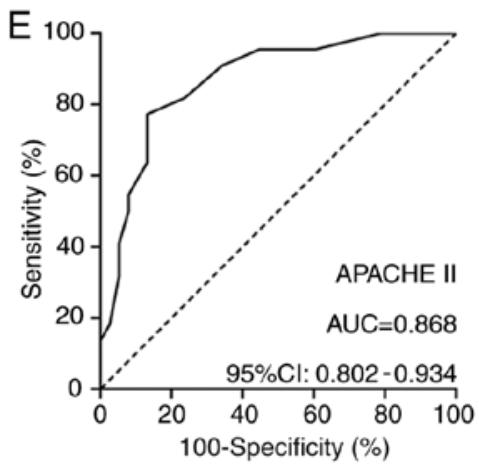

Figure 4. Relative expression and predictive value of MALAT1 plasma levels in distinguishing non-survivors from survivors. (A) MALAT1 expression was significantly increased in non-survivors compared with survivors. (B-E) Receiver operating characteristic curves for (B) MALAT1 and (C) Lac levels, (D) SOFA and (E) APACHE II scores. APACHE II, acute physiology and chronic health evaluation II; CI, confidence interval; Lac, lactate; MALAT1, metastasis-associated lung adenocarcinoma transcript 1; PCT, procalcitonin; SOFA, sepsis-related organ failure assessment.

The diagnostic and prognostic value of MALAT1 has been investigated in various types of cancer and diabetic retinopathy $(27,38)$. Recently, two studies reported the predictive values of the plasma lncRNAs nuclear enriched abundant transcript 1 and intersectin 1-2 for the diagnosis and prognosis of sepsis $(39,40)$. In the present study, multivariate logistic regression analysis revealed that MALAT1 was an independent risk biomarker for the diagnosis, severity and poor prognosis of sepsis. The results of the ROC curve analysis revealed significant predictive value for MALAT1 in differentiating patients with sepsis from HCs, patients with septic shock from those without septic shock, and non-survivors from survivors. These results indicated that MALAT1 might serve as a biomarker for the diagnosis, severity and prognosis of sepsis. It could be speculated that MALAT1 may regulate the inflammatory response to infection by targeting various genes and signaling pathways, inducing the development and progression of sepsis and increasing the risk of mortality.

There were certain limitations in this study. Firstly, this study was performed in a single center and the sample size was relatively small. Secondly, as a diagnostic biomarker, only the predictive value of MALAT1 in the diagnosis of sepsis was evaluated, whereas detailed mechanistic studies were not performed. The role and mechanism of MALAT1 in the pathogenesis of sepsis require further investigation. Thirdly, the 28-day follow-up period was relatively short, and MALAT1 expression was measured only once for each 
participant. A longer follow-up period, in addition to the continuous measurements of MALAT1, may yield more persuasive results regarding MALAT1 and sepsis-associated mortality. Finally, a systemic inflammatory response syndrome (SIRS) category was not set in this study. Sepsis was initially defined as an infection with at least two of the four SIRS criteria (41); since SIRS criteria do not reflect poor prognosis, sepsis is currently defined as a dysregulated host response to infection with a SOFA score $\geq 2$ points (2). The emphasis of this new definition is placed on mortality prediction rather than early diagnosis. Thus, the distinction between sepsis and SIRS still presents difficulty in early diagnosis of sepsis. Further mechanistic studies are necessary to determine whether MALAT1 may be a useful biomarker in distinguishing sepsis from SIRS.

In conclusion, the results of the present study demonstrated that plasma MALAT1 was upregulated in patients with sepsis, and may serve as a potential biomarker for the diagnosis, severity and prognosis of sepsis. Further investigations with larger sample sizes from multiple centers and detailed mechanistic studies are required to evaluate the clinical application of MALAT1 as a biomarker and therapeutic target for sepsis.

\section{Acknowledgements}

The authors would like to thank Dr Yuan Xue and Dr Xinxin Li (Department of Intensive Care Medicine, The First People's Hospital of Yancheng) for their technical assistance in plasma/serum samples preparation.

\section{Funding}

No funding was received.

\section{Availability of data and materials}

The datasets used and/or analyzed during the present study are available from the corresponding author on reasonable request.

\section{Authors' contributions}

LS and JC designed and coordinated the study. JC, YH and YD collected the samples and data. LS, LZ and YD performed the statistical analyses and produced graphs. YH and LZ drafted the manuscript. LS and YD revised the manuscript. All authors read and approved the final manuscript.

\section{Ethics approval and consent to participate}

This study was approved by the Ethics Committee of the First People's Hospital of Yancheng and complied with the ethics standards of the Declaration of Helsinki. All participants or their statutory guardians provided written informed consent. All identifying information of each participant was removed from this study.

\section{Patient consent for publication}

Not applicable.

\section{Competing interests}

The authors declare that they have no competing interests.

\section{References}

1. Rhodes A, Evans LE, Alhazzani W, Levy MM, Antonelli M, Ferrer R, Kumar A, Sevransky JE, Sprung CL, Nunnally ME, et al: Surviving sepsis campaign: International guidelines for management of sepsis and septic shock: 2016. Intensive Care Med 43: 304-377, 2017.

2. Singer M, Deutschman CS, Seymour CW, Shankar-Hari M, Annane D, Bauer M, Bellomo R, Bernard GR, Chiche JD, Coopersmith CM, et al: The third international consensus definitions for sepsis and septic shock (Sepsis-3). JAMA 315: 801-810, 2016.

3. Fleischmann C, Scherag A, Adhikari NK, Hartog CS, Tsaganos T, Schlattmann P, Angus DC and Reinhart K; International Forum of Acute Care Trialists: Assessment of global incidence and mortality of hospital-treated sepsis. Current estimates and limitations. Am J Respir Crit Care Med 193: 259-272, 2016.

4. Angus DC and van der Poll T: Severe sepsis and septic shock. N Engl J Med 369: 2063, 2013.

5. Mira JC, Gentile LF, Mathias BJ, Efron PA, Brakenridge SC, Mohr AM, Moore FA and Moldawer LL: Sepsis pathophysiology, chronic critical illness, and persistent inflammation-immunosuppression and catabolism syndrome. Crit Care Med 45: 253-262, 2017.

6. van der Poll T, van de Veerdonk FL, Scicluna BP and Netea MG: The immunopathology of sepsis and potential therapeutic targets. Nat Rev Immunol 17: 407-420, 2017.

7. Rivers E, Nguyen B, Havstad S, Ressler J, Muzzin A, Knoblich B, Peterson E and Tomlanovich M; Early Goal-Directed Therapy Collaborative Group: Early goal-directed therapy in the treatment of severe sepsis and septic shock. N Engl J Med 345: 1368-1377, 2001.

8. Godinjak A, Iglica A, Rama A, Tančica I, Jusufović S, Ajanović A and Kukuljac A: Predictive value of SAPS II and APACHE II scoring systems for patient outcome in a medical intensive care unit. Acta Med Acad 45: 97-103, 2016.

9. Iyer MK, Niknafs YS, Malik R, Singhal U, Sahu A, Hosono Y, Barrette TR, Prensner JR, Evans JR, Zhao S, et al: The landscape of long noncoding RNAs in the human transcriptome. Nat Genet 47: 199-208, 2015.

10. Ponting CP, Oliver PL and Reik W: Evolution and functions of long noncoding RNAs. Cell 136: 629-641, 2009.

11. Ji P, Diederichs S, Wang W, Böing S, Metzger R, Schneider PM, Tidow N, Brandt B, Buerger H, Bulk E, et al: MALAT-1, a novel noncoding RNA, and thymosin beta4 predict metastasis and survival in early-stage non-small cell lung cancer. Oncogene 22: 8031-8041, 2003.

12. Zhang X, Hamblin MH and Yin KJ: The long noncoding RNA Malat1: Its physiological and pathophysiological functions. RNA Biol 14: 1705-1714, 2017.

13. Lipovich L, Dachet F, Cai J, Bagla S, Balan K, Jia H and Loeb JA: Activity-dependent human brain coding/noncoding gene regulatory networks. Genetics 192: 1133-1148, 2012.

14. Watts R, Johnsen VL, Shearer J and Hittel DS: Myostatin-induced inhibition of the long noncoding RNA Malat1 is associated with decreased myogenesis. Am J Physiol Cell Physiol 304: C995-C1001, 2013.

15. Michalik KM, You X, Manavski Y, Doddaballapur A, Zörnig M, Braun T, John D, Ponomareva Y, Chen W, Uchida S, et al: Long noncoding RNA MALAT1 regulates endothelial cell function and vessel growth. Circ Res 114: 1389-1397, 2014.

16. Yoshimoto R, Mayeda A, Yoshida M and Nakagawa S: MALAT1 long non-coding RNA in cancer. Biochim Biophys Acta 1859: 192-199, 2016.

17. Uchida S and Dimmeler S: Long noncoding RNAs in cardiovascular diseases. Circ Res 116: 737-750, 2015.

18. Zhang $\mathrm{X}$, Tang $\mathrm{X}$, Liu $\mathrm{K}$, Hamblin $\mathrm{MH}$ and Yin KJ: Long noncoding RNA malat1 regulates cerebrovascular pathologies in ischemic stroke. J Neurosci 37: 1797-1806, 2017.

19. Vergadi E, Vaporidi K and Tsatsanis C: Regulation of endotoxin tolerance and compensatory anti-inflammatory response syndrome by Non-coding RNAs. Front Immunol 9: 2705, 2018.

20. Yang H, Liang N, Wang M, Fei Y, Sun J, Li Z, Xu Y, Guo C, Cao Z, Li S and Jiao Y: Long noncoding RNA MALAT-1 is a novel inflammatory regulator in human systemic lupus erythematosus. Oncotarget 8: 77400-77406, 2017. 
21. Shaker OG, Mahmoud RH, Abdelaleem OO, Ibrahem EG, Mohamed AA, Zaki OM, Abdelghaffar NK, Ahmed TI, Hemeda NF, Ahmed NA and Mansour DF: LncRNAs, MALAT1 and lnc-DC as potential biomarkers for multiple sclerosis diagnosis. Biosci Rep 39: BSR20181335, 2019.

22. Pan F, Zhu L, Lv H and Pei C: Quercetin promotes the apoptosis of fibroblast-like synoviocytes in rheumatoid arthritis by upregulating lncRNA MALAT1. Int J Mol Med 38: 1507-1514, 2016.

23. Livak KJ and Schmittgen TD: Analysis of relative gene expression data using real-time quantitative PCR and the 2(-Delta Delta C(T)) method. Methods 25: 402-408, 2001.

24. Tano K, Mizuno R, Okada T, Rakwal R, Shibato J, Masuo Y, Ijiri K and Akimitsu N: MALAT-1 enhances cell motility of lung adenocarcinoma cells by influencing the expression of motility-related genes. FEBS Lett 584: 4575-4580, 2010.

25. Huang C, Han J, Wu Y, Li S, Wang Q, Lin W and Zhu J: Exosomal MALAT1 derived from oxidized low-density lipoprotein-treated endothelial cells promotes M2 macrophage polarization. Mo Med Rep 18: 509-515, 2018.

26. Gao F, Chen R, Xi Y, Zhao Q and Gao H: Long noncoding RNA MALAT1 regulates sepsis in patients with burns by modulating miR-214 with TLR5. Mol Med Rep 19: 3756-3766, 2019.

27. Shaker OG, Abdelaleem OO, Mahmoud RH, Abdelghaffar NK, Ahmed TI, Said OM and Zaki OM: Diagnostic and prognostic role of serum miR-20b, miR-17-3p, HOTAIR, and MALAT1 in diabetic retinopathy. IUBMB Life 71: 310-320, 2019.

28. Zhang B, Arun G, Mao YS, Lazar Z, Hung G, Bhattacharjee G, Xiao X, Booth CJ, Wu J, Zhang C and Spector DL: The lncRNA Malat 1 is dispensable for mouse development but its transcription plays a cis-regulatory role in the adult. Cell Rep 2: 111-123, 2012

29. Chen H, Wang $X$, Yan $X$, Cheng $X, H e X$ and Zheng W: LncRNA MALAT1 regulates sepsis-induced cardiac inflammation and dysfunction via interaction with miR-125b and p38 MAPK/NFkB. Int Immunopharmacol 55: 69-76, 2018.

30. Yu Z, Rayile A, Zhang X, Li Y and Zhao Q: Ulinastatin protects against lipopolysaccharide-induced cardiac microvascular endothelial cell dysfunction via downregulation of lncRNA MALAT1 and EZH2 in sepsis. Int J Mol Med 39: 1269-1276, 2017.

31. Dai L, Zhang G, Cheng Z, Wang X, Jia L, Jing X, Wang H, Zhang R, Liu M, Jiang T, et al: Knockdown of LncRNA MALAT contributes to the suppression of inflammatory responses by up-regulating miR-146a in LPS-induced acute lung injury. Connect Tissue Res 59: 581-592, 2018.
32. Ding Y, Guo F, Zhu T, Li J, Gu D, Jiang W, Lu Y and Zhou D: Mechanism of long non-coding RNA MALAT1 in lipopolysaccharide-induced acute kidney injury is mediated by the miR-146a/NF- $\mathrm{BB}$ signaling pathway. Int J Mol Med 41: 446-454, 2018.

33. Pan L, Liu D, Zhao L, Wang L, Xin M and Li X: Long noncoding RNA MALAT1 alleviates lipopolysaccharide-induced inflammatory injury by upregulating microRNA-19b in murine chondrogenic ATDC5 cells. J Cell Biochem 119: 10165-10175, 2018.

34. Gast M, Rauch BH, Nakagawa S, Haghikia A, Jasina A, Haas J, Nath N, Jensen L, Stroux A, Böhm A, et al: Immune system-mediated atherosclerosis caused by deficiency of long non-coding RNA MALAT1 in ApoE-/-mice. Cardiovasc Res 115: 302-314, 2019.

35. Zhao G, Su Z, Song D, Mao Y and Mao X: The long noncoding RNA MALAT1 regulates the lipopolysaccharide-induced inflammatory response through its interaction with $\mathrm{NF}-\kappa \mathrm{B}$. FEBS Lett 590: 2884-2895, 2016.

36. Castelli GP, Pognani C, Cita M, Stuani A, Sgarbi L and Paladini R: Procalcitonin, C-reactive protein, white blood cells and SOFA score in ICU: Diagnosis and monitoring of sepsis. Minerva Anestesiol 72: 69-80, 2006.

37. Dahaba AA and Metzler H: Procalcitonin's role in the sepsis cascade. Is procalcitonin a sepsis marker or mediator? Minerva Anestesiol 75: 447-452, 2009

38. Tian X and Xu G: Clinical value of lncRNA MALAT1 as a prognostic marker in human cancer: Systematic review and meta-analysis. BMJ Open 5: e008653, 2015.

39. Zeng Q, Wu J and Yang S: Circulating lncRNA ITSN1-2 is upregulated, and its high expression correlates with increased disease severity, elevated inflammation, and poor survival in sepsis patients. J Clin Lab Anal 33: e22836, 2019.

40. Huang Q, Huang C, Luo Y, He F and Zhang R: Circulating lncRNA NEAT1 correlates with increased risk, elevated severity and unfavorable prognosis in sepsis patients. Am J Emerg Med 36: 1659-1663, 2018.

41. American college of chest physicians/society of critical care medicine consensus conference: Definitions for sepsis and organ failure and guidelines for the use of innovative therapies in sepsis. Crit Care Med 20: 864-874, 1992. 